\title{
Beclin 1 cleavage by caspase-3 inactivates autophagy and promotes apoptosis
}

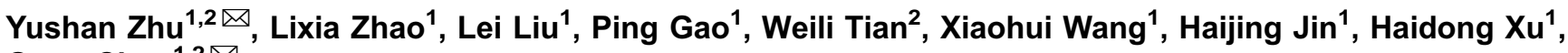 \\ Quan Chen ${ }^{1,2} \bowtie$ \\ ${ }^{1}$ The Joint Laboratory of Apoptosis and Cancer Biology, College of Life Sciences, Nankai University, Tianjin 300071, China \\ 2 The National Key Laboratory of Biomembrane and Membrane Biotechnology, Institute of zoology, Chinese Academy of \\ Sciences, Beijing 100101, China
}

冈 Correspondence: chenquan@nankai.edu.cn (Q. C), zhuys@nankai.edu.cn (Y. Z)

Received March 24, 2010 Accepted April 14, 2010

\begin{abstract}
Autophagy and apoptosis are both highly regulated biological processes that play essential roles in tissue homeostasis, development and diseases. Autophagy is also described as a mechanism of death pathways, however, the precise mechanism of how autophagy links to cell death remains to be fully understood. Beclin 1 is a dual regulator for both autophagy and apoptosis. In this study we found that Beclin 1 was a substrate of caspase- 3 with two cleavage sites at positions 124 and 149, respectively. Furthermore, the autophagosome formation occurred, followed by the appearance of morphological hallmarks of apoptosis after staurosporine treatment. The cleavage products of Beclin 1 reduced autophagy and promoted apoptosis in HeLa cells and the cells in which Beclin 1 was stably knocked down by specific shRNA. In addition, the cleavage of Beclin 1 resulted in abrogating the interaction between $\mathrm{Bcl}-2$ with Beclin 1, which could be blocked by z-VAD-fmk. Thus, our results suggest that the cleavage of Beclin 1 by caspase-3 may contribute to inactivate autophagy leading towards augmented apoptosis.
\end{abstract}

KEYWORDS beclin 1, autophagy, apoptosis, caspase cleavage

\section{INTRODUCTION}

Autophagy is a genetically programmed process that degrades long-lived cellular proteins and organelles. In response to nutrient-limiting conditions or environmental insults (Levine and Kroemer, 2008; Orvedahl and Levine, 2008; Levine and Kroemer, 2009), autophagy is activated to form a double-membrane autophagosome. Then the autophagosomes mature by fusing with endosomes and lysosomes for the degradation of the cargo (Kroemer and Jaattela, 2005; Yorimitsu and Klionsky, 2005). In the case of starvation and withdrawal of growth factors, autophagy is activated to generate ATP and nutrients through limited self-digestion to maintain vital cellular functions. Autophagy also serves as a cell survival mechanism by removing the damaged mitochondria and other organelles, misfolded protein aggregates and intracellular pathogens. On the other hand, autophagy has recently been considered as a mechanism of death pathways, or autophagic cell death, through excessive selfdigestion and degradation of essential cellular components (Codogno and Meijer, 2005; Kroemer and Jaattela, 2005; Maiuri et al., 2007c; Eisenberg-Lerner et al., 2009). Autophagic cell death occurs in both caspase dependent and caspase independent manners. Despite intensive studies, autophagic cell death is mostly evaluated by morphological criteria and morphological features, and the precise mechanism of how autophagy links to cell death remains to be fully understood.

Beclin 1, a novel BH3-only protein, is a key component of a Class III PI3K complex involved in the initiation of autophagosome formation. It has recently been reported that Beclin 1 interacts with Bcl-2 and Bcl-xL (Maiuri et al., 2007b; Oberstein et al., 2007) to inhibit autophagy (Shimizu et al., 2004; Pattingre et al., 2005; Pattingre and Levine, 2006). Sequential, structural and functional analyses have revealed that Beclin 1, similar to other proapoptotic molecules such as Bak, Bad and Bim, contains a $\mathrm{BH} 3$ domain from 108 to 127 
residues, which is responsible for its binding with $\mathrm{Bcl}-2$ and Bcl-xL (Feng et al., 2007; Maiuri et al., 2007b; Oberstein et al., 2007). Several amino acids in this region including L112, L116, G120 and F123 are buried in the hydrophobic groove of $\mathrm{Bcl}-\mathrm{xL}, \mathrm{D} 121$, another amino acid in the groove, forms ion pairs with a conserved arginine from Bcl-xL (Pattingre et al., 2005; Maiuri et al., 2007a, b). It is believed that several proapoptotic $\mathrm{Bcl}-2$ family members including Bax, Bak, Bad, and $\mathrm{ABT737}$, a BH3-mimetic compound, promote autophagy via competitively disrupting the interaction between Beclin 1 and Bcl-2/Bcl-xL (Maiuri et al., 2007a). The crosstalk of autophagy and apoptosis could also be regulated by JNK which phosphorylates Bcl-2 (Wei et al., 2008a; Pattingre etal., 2009). The starvation-indcued activation of JNK1 can cause multi-site phosphorylation in the between $\mathrm{BH} 4$ and $\mathrm{BH} 3$ domain of $\mathrm{Bcl}-2$, which leads to disrupt the interaction of $\mathrm{Bcl}-2$ and Beclin 1 (Wei et al., 2008a, b; Pattingre et al., 2009). Obviously Beclin 1 interacting with the Bcl-2 family members is likely the convergent point for autophagic and apoptotic machinery (Wang, 2008). In this study, we demonstrate that Beclin 1 is cleaved with two cleavage sites by caspase-3 during staurosporine-induced apoptosis, which promotes apoptosis in apoptotic competent cells through inactivating autophagy.

\section{RESULTS}

\section{Staurosporine induced early autophagy followed by apoptosis}

To understand the relationship between autophagy and apoptosis, we first examined the occurrence of autophagosome formation during apoptosis induced by staurosporine. Autophagy was measured by the formation of autophagosomes as indicated by GFP-LC3 redistribution from a diffuse staining pattern to a cytoplasmic punctuate structure. We found that the number of autophagosomes in the cells was dramatically increased at $3 \mathrm{~h}$ and then started to be decreased at $12 \mathrm{~h}$ after the treatment of GFP-LC3/HeLa cells with staurosporine (Fig. $1 \mathrm{~A}$ and $1 \mathrm{~B}$ ). Also, there was a progressive increase of LC3-II from 3 to $12 \mathrm{~h}$ and then decreased at $18 \mathrm{~h}$ as detected by Western blotting (Fig. 1C). The reduction of autophagosome formation and LC3-II levels could be inhibited by Bafilomycin A1 (BA1, $20 \mathrm{nM}$ ), which is prevent the fusion between lysosomes and autophagosomes. Interestingly, the disappearance of autophagosomes and reduced levels of LC3-II were correlated with an increase in apoptosis (Fig. 1 and Supplemental Fig. 1). The apoptotic hallmarks were evident as measured by annexin V/PI staining (Fig. 1D and 1E) and caspase activation along with the decreasing of autophagosomes and LC3 (Fig. 2A and 2B). Other death stimuli such as TRAIL and PAO (phenylarsine oxide) also induced an early increase of autophagy which was followed by apoptosis in the cells (data not shown). Cell death was inhibited and the process of autophagy was significantly prolonged when caspase activity was blocked by z-VAD-fmk (Fig. 1A, 1B and 2B). EBSS was used as a positive control for autophagy without induction of cell death.

\section{Beclin 1 is cleaved by caspase-3 at aa 124 and 149 sites}

We next examined the expression of Beclin 1 during the apoptosis. Interestingly, we found the cleaved products of Beclin 1 started to be shown up in $3 \mathrm{~h}$ after the cells were treated with staurosporine. At the same time, caspase- 3 activation was detectable as measured by Western blotting and flow cytometric analysis. However, the cleavage could be completely inhibited in the cells overexpressing Bcl-2 (Fig. 2A and $2 B$ ). To test whether Beclin 1 could be directly cleaved by the activated caspase-3, Beclin 1/myc was expressed and incubated with purified and activated caspase-3. As expected, caspase- 3 cleaved Beclin 1 into two distinct products, which could be completely inhibited by Z-VAD-fmk (Fig. 2C). Sequence analysis revealed that Beclin 1 has two typical putative caspase cleavage sites at positions 124 and 149 (Fig. 2C). To determine the cleavage sites of Beclin 1, we substituted, individually and together, the consensus asparagines with alanines at positions 124 and 149 of Beclin (D124A, D149A and D124/129A), and analyzed the effects of these mutations on the cleavage of Beclin 1 by caspase- 3 in vitro and in vivo. Interestingly, activated caspase- 3 was able to cleave either of D124A and D149A, but not to D124/149A, confirming that both positions 124 and 149 on Beclin 1 are caspase 3 cleaving sites (Fig. $2 \mathrm{C}$ ). This result was further confirmed by expressing wild-type and the D124/149A double mutant in HeLa cells. The D124/149A mutant was completely resistant to the cleavage during staurosporineinduced apoptosis (Fig. 2D). These results demonstrate that caspase-3 cleaved Beclin 1 at positions 124 and 149, which could be inhibited by both pharmacological inhibitor Z-VADfmk and Bcl-2.

\section{Beclin 1 mutants inhibit cell autophagy}

To address the functional consequence of Beclin 1 cleavage, we ectopically expressed the cleaved products (D1-149 and D150-450) of Beclin 1 in HeLa cells. Intriguingly, the autophagosomes formation was dramatically increased in the cells expressing wild-type Beclin 1 in response to starvation (Fig. $3 \mathrm{~A}$ and $3 \mathrm{~B}$ ) or staurosporine (Fig. 3D and $3 \mathrm{E}$, Supplemental Fig. 2). In contrast, the number of autophagosomes was significantly reduced in the cells expressing other mutants or truncates under the same condition. Furthermore, a large portion of LC3-I was converted to LC3-II in the cells expressing wild type Beclin 1, but much less in the cells expressing the noncleavable mutant and either D1-149 or D150-450 (Fig. 3C and 3E). The cells 
A
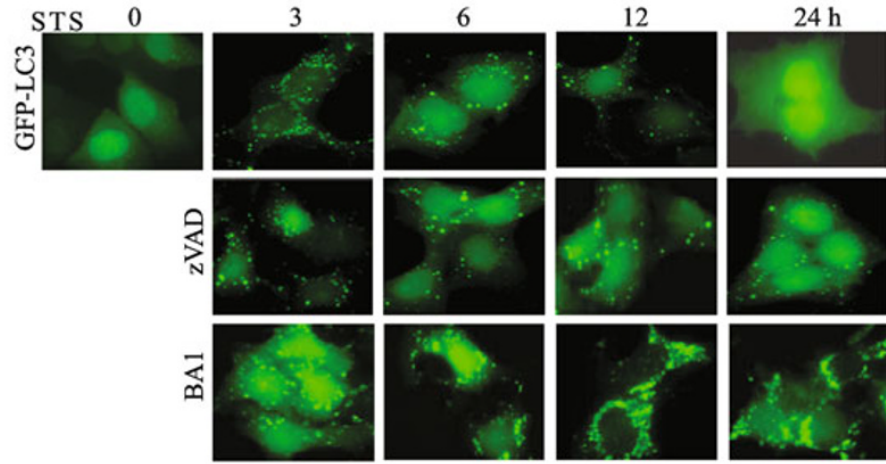

D
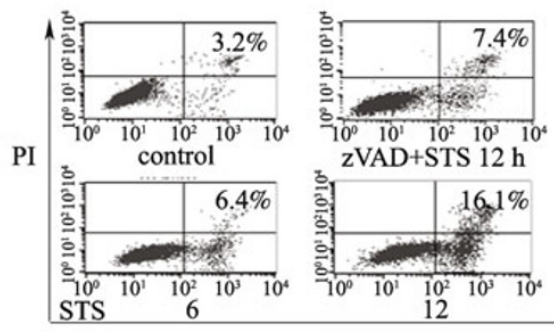
zVAD+STS $12 \mathrm{~h}$

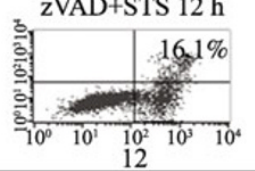

FITC-Annexin V
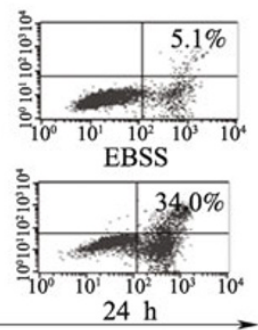

$24 \mathrm{~h}$
B

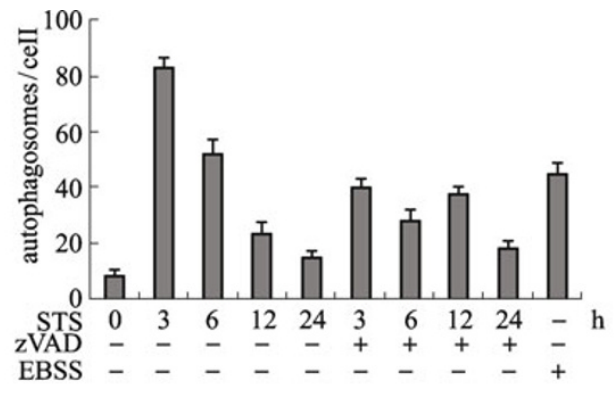

C

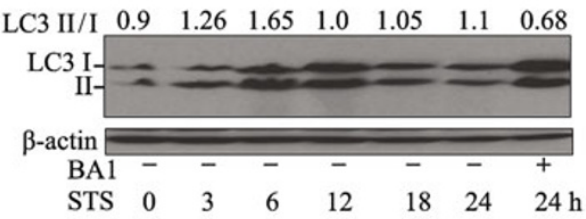

E

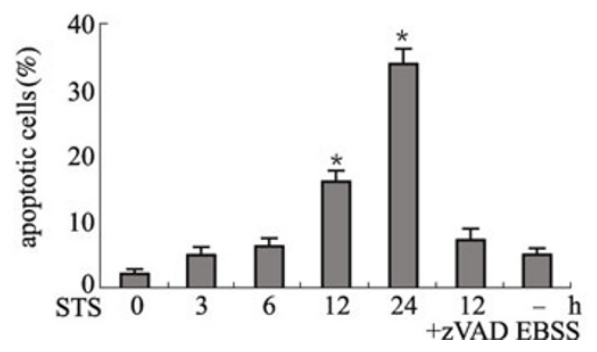

Figure 1. Staurosporine induced cell death and autophagy. (A) Autophagosome formation in response to staurosporine. GFPLC3 stably expressing HeLa cells were cultured on glass coverslips and then treated by staurosporine $(0.1 \mu \mathrm{M})$ in the presence or absence of $z-V A D$ or BA1, respectively, at the indicated times and then fixed with formaldehyde $(3.7 \%, w / v)$ for autophagic assays. GFP-LC3 redistribution from a diffuse staining pattern in the cytoplasm and nucleus to a cytoplasmic punctuate structure was used to measure autophagy as described in the Materials and Methods. (B) The statistical results of autophagosome formation following treatment with staurosporine and in the presence or absence of Z-VAD $(50 \mu \mathrm{M})$, and EBSS was used as positive control. All the data were the mean value of at least 3 independent experiments. (C) Western blotting analysis of the LC3-II levels following treatment with staurosporine as indicated time in the presence or absence of BA1 $(20 \mathrm{nM})$. (D) Measurement of apoptosis induced by staurosporine. Apoptosis was analyzed by flow cytometric analysis after FITC-Annexin V/PI staining as described in the Methods. Cells were treated with staurosporine $(0.1 \mu \mathrm{M})$ for $0,6,12,24 \mathrm{~h}$ and EBSS for $2 \mathrm{~h}$, respectively. (E) The statistical results of apoptosis were obtained from both right hand quadrants (both the $\mathrm{PI}$ and Annexin $\mathrm{V}$ positive cells). All the data were the mean value of at least 3 independent experiments. ${ }^{*} p<0.05$ compared with the control cells.

overexpressing Bcl-2 was used as a negative control. These results clearly show that the cleavage of Beclin 1 resulted in inactivating autophagy.

\section{Beclin 1 mutants increase the sensitivity of cell death}

To determine the effect of the cleavage of Beclin 1 by caspase- 3 on cell apoptosis, we tested the sensitivity of HeLa cells expressing these mutants to staurosporine. As shown in Fig. 4, the activation of caspase and cell death are significantly increased in the cells expressing the truncated forms of Beclin 1 when compared to that in the cells expressing wild-type Beclin 1 after treatment with staurosporine (Fig. 4), suggesting that the caspase-3 cleavage of Beclin 1 promotes cell death and inhibits autophagy. It is interesting to note that the cleaved products of Beclin 1 promoting apoptosis are the putative $\mathrm{BH}-3$ domain independent. This raised a question that the interaction between Beclin1 and Bcl-2 may determine their biologic effects (Maiuri et al., 2007a).

\section{The cleavage of Beclin 1 disrupts its interaction with Bcl-2}

The interaction of Beclin 1 with $\mathrm{Bcl}-2$ is suggested to play a regulatory role in autophagy (Pattingre et al., 2005). It is therefore of interest to check if the cleavage of Beclin 1 by caspase-3 could affect the interaction between Bcl-2 and Beclin 1. As shown in Fig. 5, we found that the interaction was almost completely disrupted once apoptosis is starting (12 $\mathrm{h}$ ) during staurosporine treatment. Importantly, in the presence of z-VAD-fmk, the dissociation of Beclin 1 from Bcl-2 was largely blocked. This strongly suggests that the cleavage of Beclin 1 may cause the dissociation of Beclin 1 from Bcl-2. To 
A

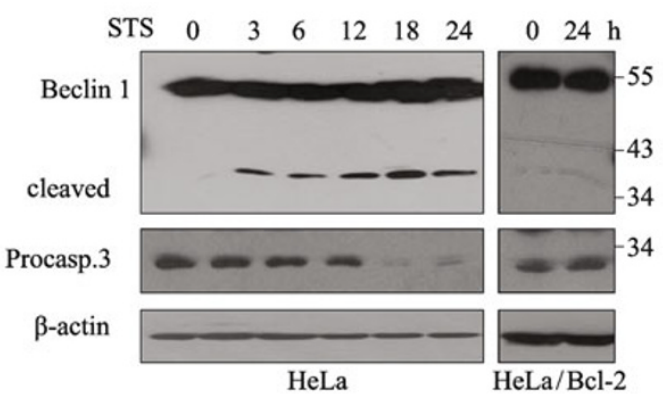

$\mathrm{C}$

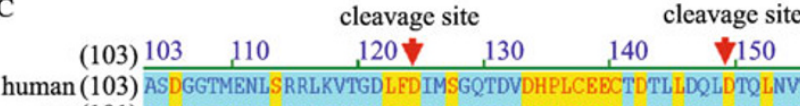
rat (101) ASDGGTMENLSRRLKVTGDLFDIMSGQTDVDHPLCEECTDTLLDQLDTQLNV Bos taurus (101) ASDGGTMENLSRRLKVTGDLFDIMSGQTDVDHPLCEECTDTLLDQLDTQLNV Drosophila (77) GRDN---TKMSAAEN LKABLFDCLSSNSEIDHPLCEECADSMLBIMDRELPI: consensus (103) ASDGGTMENLSRRLKVTGDLFDIMSGQTDVDHPLCEECTDTLLDQLDTQLNV] $\mathrm{BH} 3$ domain

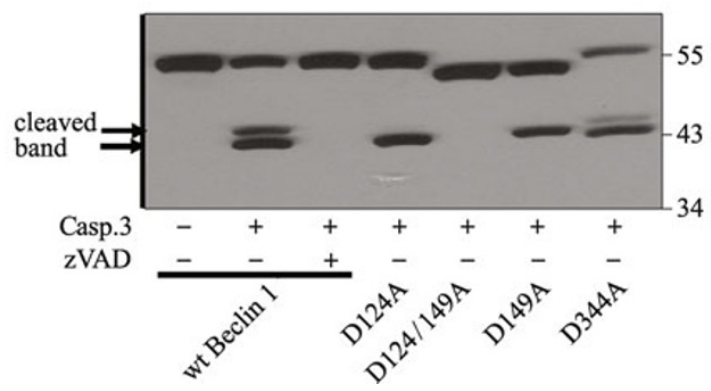

B

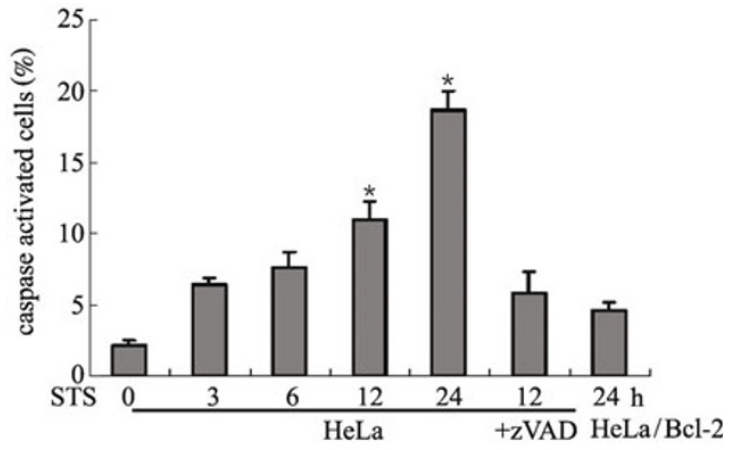

D

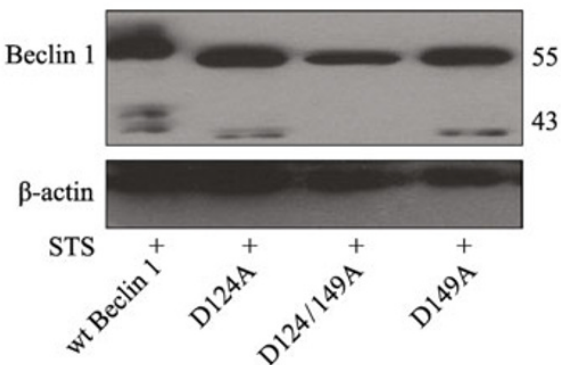

Figure 2. Beclin 1 is cleaved at two distinct sites by caspase-3. (A) Western blotting analysis detected Beclin 1 expression following the treatment at the indicated times with staurosporine $(0.1 \mu \mathrm{M})$ in HeLa cells and $\mathrm{Bcl}-2$ overexpressing HeLa cells. (B) Caspase activity assay by FITC-VAD-fmk following treatment with staurosporine $(0.1 \mu \mathrm{M})$ in HeLa cells and Bcl-2 overexpression cells. ${ }^{*} p<0.05$ compared with the control cells. (C) Sequence alignment of the evolutionally conserved amino acid of Beclin 1 from several species. Residues that are identical and similar among the Beclin 1 are denoted in yellow and blue, respectively. The mutation sites are indicated by arrows (up). The Beclin 1 cleavage sites were confirmed by Western blotting (down). Wild-type Beclin 1, D124A, D149A, D124/149A and D344A mutants were incubated with activated recombinant caspase-3 in vitro. $20 \mu \mathrm{g}$ of cell lysates were incubated with $200 \mathrm{ng}$ of recombinant caspase- 3 at $37^{\circ} \mathrm{C}$ for $2 \mathrm{~h}$ in $50 \mu \mathrm{L}$ of reaction buffer $(100 \mathrm{mM} \mathrm{Hepes} \mathrm{pH} 7.5,20 \%$ glycerol, $0.5 \mathrm{mM}$ EDTA, $10 \mathrm{mM} \mathrm{DTT}$ ) in the presence or absence of z-VAD-fmk. The reactions were stopped with $10 \mu \mathrm{L} 6 \times$ loading buffer and boiled for $5 \mathrm{~min}$ and then resolved by $12 \%$ SDS-PAGE and Western blotted with anti-Beclin 1 antibody. (D) Mutation at two cleavage sites of Beclin 1 prevents its cleavage in staurosporine treated cells. HeLa cells expressing Beclin 1, D124/149A, D124A and D149A were treated by staurosporine for $24 \mathrm{~h}$ and then collected for Western blotting by anti-Beclin 1 antibody.

further confirm the observation, we examined the interaction between $\mathrm{Bcl}-2$ and Beclin 1 mutants, and revealed that although D150-450 remains to weakly interact with $\mathrm{Bcl}-2$, the interaction between $\mathrm{Bcl}-2$ and $\mathrm{D} 1-149$ was vanished (Fig. 5B). Unexpectedly, the noncleavable double mutant displayed a reduced capability to interact with $\mathrm{Bcl}-2$, most probably due to the fact that the mutation of these sites may cause conformational changes to this protein as indicated by the altered gel mobility. This may explain why the noncleavable mutant is able to promote cell death and inhibits autophagy.

\section{The effects of Beclin 1 and its mutants on autophagy and apoptosis in Beclin KD cells}

To further address if the truncated forms of Beclin 1 indeed function to reduce autophagy and enhance apoptosis, we established cell lines in which Beclin 1 was specifically knocked down by shRNA. Then RNAi resistant Beclin 1 or its mutants were co-transfected with GFP-LC3 into Beclin 1 knock down (KD) cells and, the cells were then treated with $0.1 \mu \mathrm{M}$ staurosporine for $6 \mathrm{~h}$ in the presence or absence of BA1 $(20 \mathrm{nM})$. As shown in Fig. 6A, 6B and Supplemental 
A

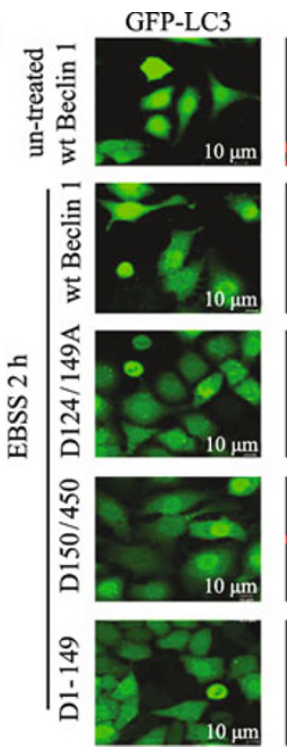

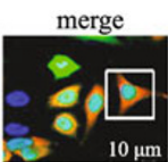
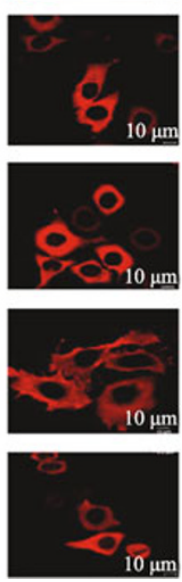
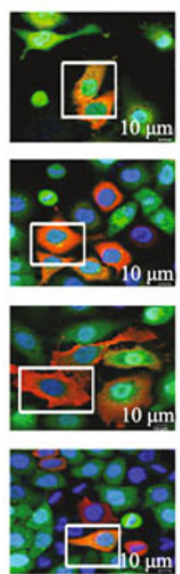
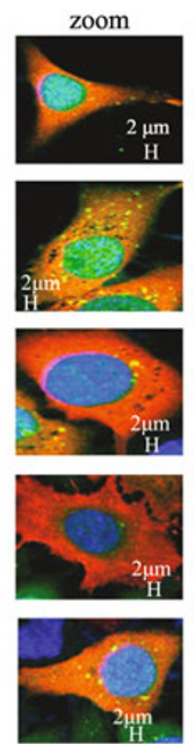

B

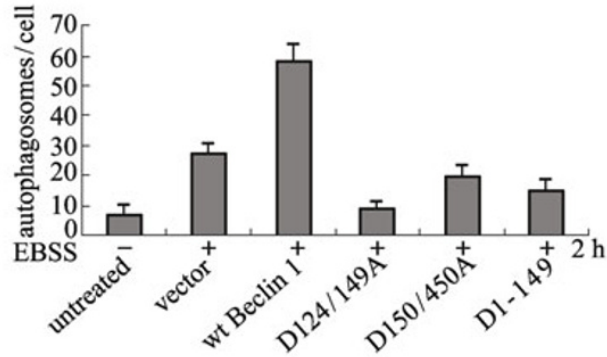

$\mathrm{C}$

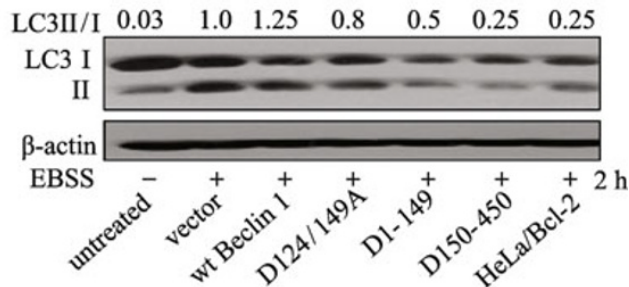

E

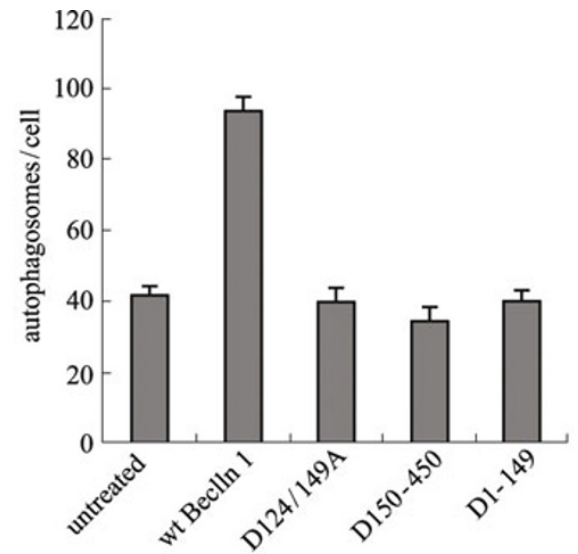

F

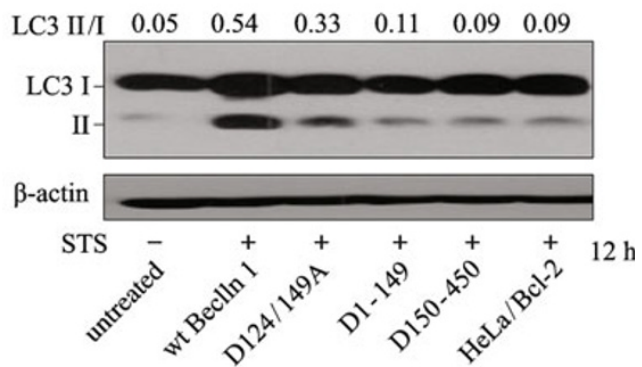

Figure 3. Beclin 1 mutants inhibit the autophagosome formation induced by EBSS or by staurosporine. (A) GFP-LC3/ HeLa cells were transfected with Beclin 1 or its mutants and then treated by EBSS for $2 \mathrm{~h}$. Cells were then immunostained with antimyc antibody. GFP-LC3 redistribution from a diffuse staining pattern in the cytoplasm and nucleus to a cytoplasmic punctuate structure was used to measure autophagy. (B) Statistical results shown in Fig. 3A and data was mean value of 3 independent experiments. (C) Cells expressing Beclin 1, its mutants or Bcl-2 were treated with EBSS for $2 \mathrm{~h}$. Western blotting analyses were performed to detect the LC3-I and LC3-II expression change. (D) Described in Fig. 3A except the by $0.1 \mu \mathrm{M}$ staurosporine for $12 \mathrm{~h}$. (E) Statistical results shown in Fig. 3D and data was mean value of 3 independent experiments. (F) Described in Fig. $3 \mathrm{C}$ except the by $0.1 \mu \mathrm{M}$ staurosporine for $12 \mathrm{~h}$ to detect the LC3-I and LC3-II expression change. 
A

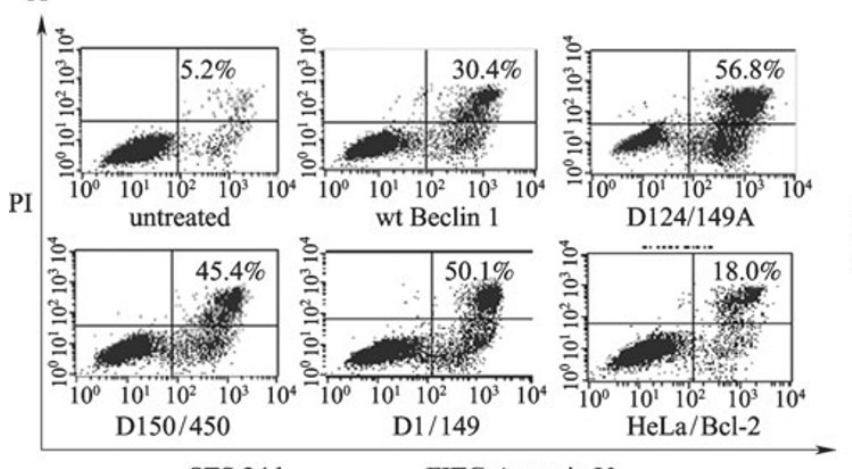

STS $24 \mathrm{~h}$

FITC-Annexin V

B

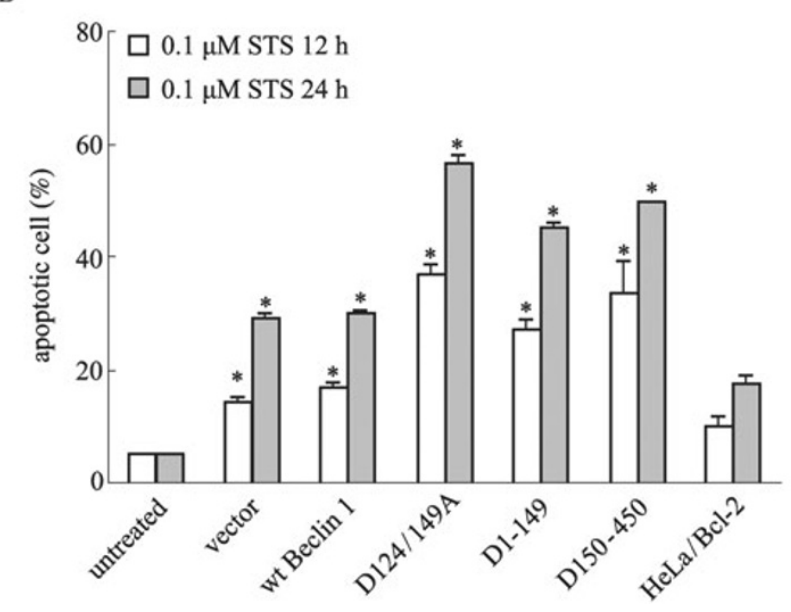

C

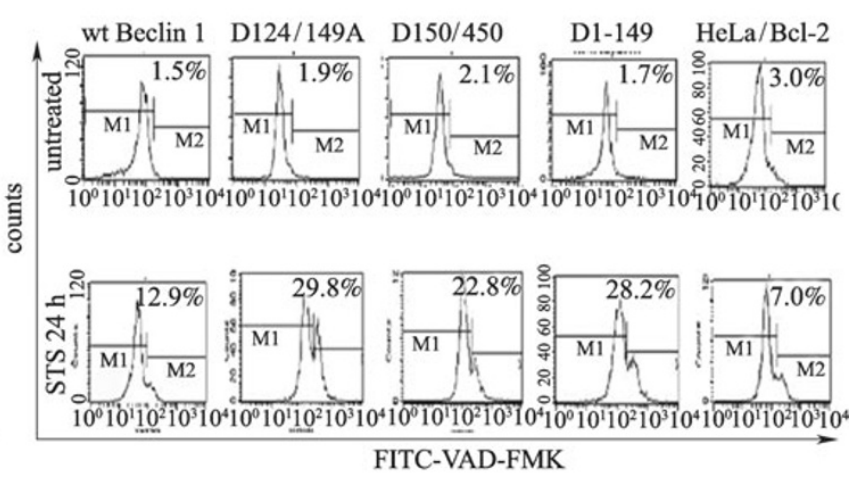

D

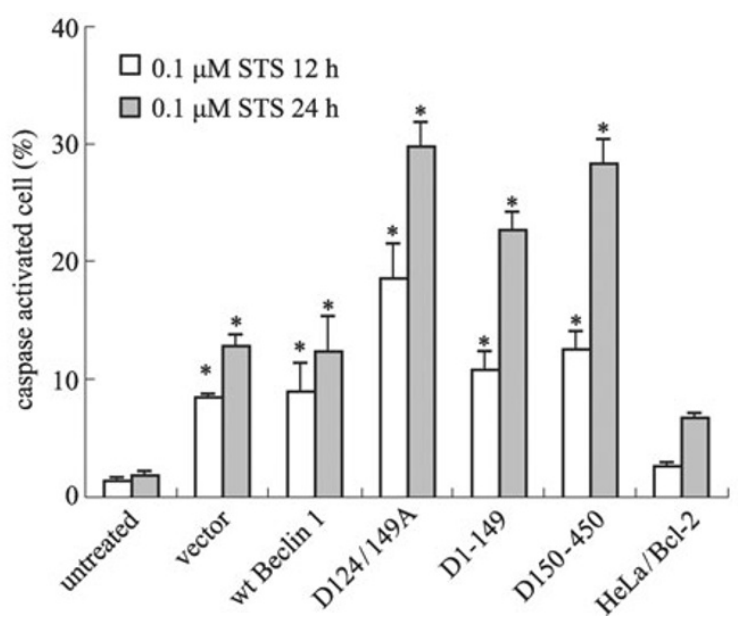

Figure 4. Beclin 1 mutants and truncations increase the sensitivity of cell death. Cells expressing Beclin 1 and its mutants or truncations were treated by staurosporine $(0.1 \mu \mathrm{M})$ for the indicated times, the percentage of apoptotic cells $(A, B)$ and caspase activated cells (C, D) were determined by FITC-Annnexin V/PI staining and FITC-VAD-fmk staining (mean \pm S.D. $n=3$ ), respectively, as described in Fig. 1D. Data were the mean value of at least 3 independent experiments. ${ }^{*} p<0.05$ compared with the control cells.

Fig. 3, the autophagosome formation under stimulation with staurosporine was remarkably reduced in Beclin $1 \mathrm{KD}$ cells, which could be restored by the reintroduction of wild type Beclin 1. Furthermore, knock down of Beclin 1 resulted in a reduced level of LC3-II form in the cells. However, the switch from LC3-I to LC3-II could be recovered by expressing an RNAi resistant wild type of Beclin 1 in the cells. The noncleavable mutant and D1-149, but not D150-450, also slightly increased the LC3-II level (Fig. 6C).

To determine the effect of mutant Beclin 1 on cell death in Beclin $1 \mathrm{KD}$ cells, we treated the cells expressing either of a RNAi resistant wild type Beclin 1, the noncleavable mutant, D1-149 and D150-450 with staurosporine. The RNAi resistant wild type Beclin 1 inhibited cell death whereas the noncleavable mutant enhanced the sensitivity to staurosporine. Interestingly, D150 450 was able to significantly increase cell death in presence or absence of staurosporine (Fig. 6D), suggesting that the cleaved fragment of Beclin 1 by caspase-3 alone may function as an effector in triggering cell death.

\section{DISCUSSION}

In this study we identified two caspase-3 cleavage sites in Beclin 1, which may be responsible for the regulation of autophagy and apoptosis. Further investigation revealed that the cleavage of Beclin 1 indeed inactivated autophagy and promoted apoptosis. Our results support the theory that autophagy is one of the initial responses for survival in apoptosis competent cells. However, if the stress is severe and sustained, the cells overcome the survival mechanism by cleaving the key component of autophagy such as Beclin 1 (Cho et al., 2009; Luo and Rubinsztein, 2009) or ATG5 (Yousefi et al., 2006), leading to apoptosis. Our data are consistent with recent reports showing that Beclin 1 cleavage reduces the level of autophagy (Cho et al., 2009; Luo and Rubinsztein, 2009), and further demonstrate that caspase-3 cleavage of Beclin 1 may regulate the switch of autophagy toward apoptosis. ATG5, another autophagic regulator, is also found to be cleaved during apoptosis, and the cleavage product appears to promote mitochondria-mediated 

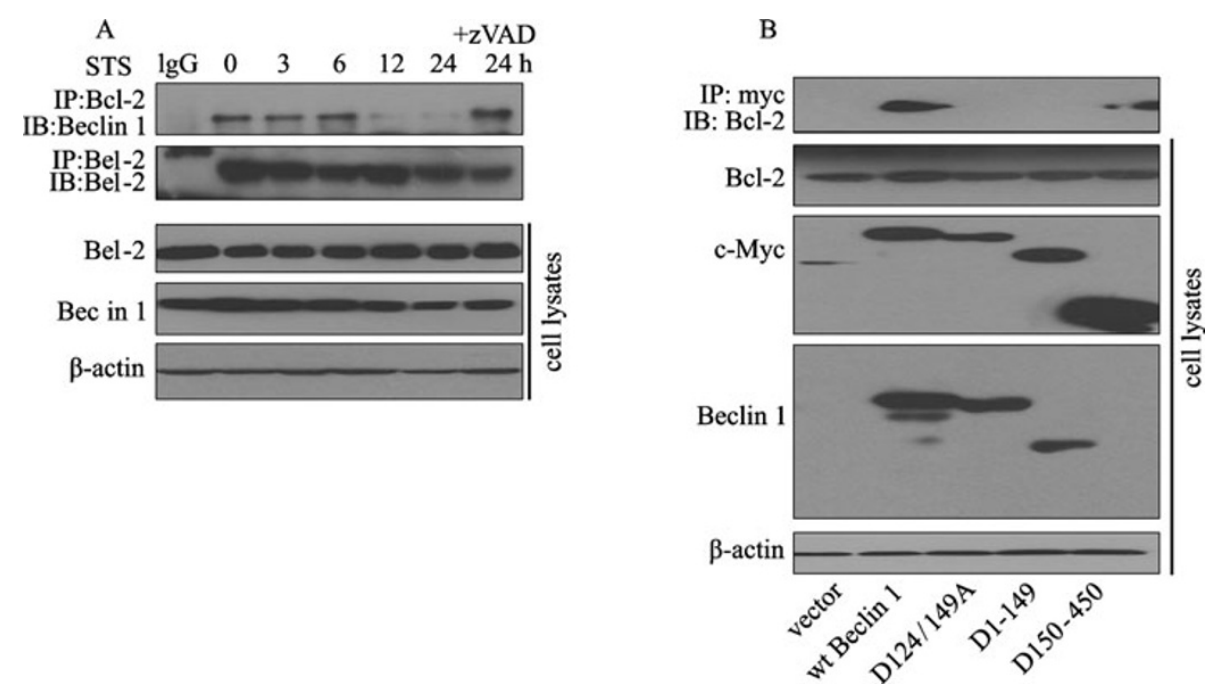

Figure 5. Interaction between Bcl-2 and Beclin 1 or its mutants and truncations. (A) The interaction of Bcl-2 with endogenous Beclin 1. HeLa cells were transfected with Bcl-2/HA-tag plasmid for $24 \mathrm{~h}$ and then treated with staurosporine $(0.1 \mu \mathrm{M})$ as the indicated times in the presence or absence of z-VAD-fmk $(50 \mu \mathrm{M})$. The cell lysates were then subjected to co-Immunoprecipitation with anti$\mathrm{Bcl}-2$ monoclonal antibody. The interactions were then analyzed by Western blotting with anti-Beclin 1 monoclonal antibody. (B) The interaction of Bcl-2 with wild-type and mutant or truncation forms of Beclin 1. Cells were co-transfected with HA-Bcl-2 and Beclin 1myc or its mutants for $24 \mathrm{~h}$ and subjected to co-immunoprecipitation with anti-myc monoclonal antibody. The interactions were analyzed by Western blotting with anti-Bcl-2 monoclonal antibody.

apoptosis (Yousefi et al., 2006). Obviously, the cleavage of Beclin 1, a key protein in autophagy, represents a critical step for inducing apoptosis.

A previous report suggests that Beclin 1 has one cleavage site at position 149 (Luo and Rubinsztein, 2009). However, we clearly demonstrate that there are two caspase-3 cleavage sites adjacent to the Beclin $1 \mathrm{BH} 3$ domain. Importantly, our result showed that the cleavage of Beclin 1 at the two sites may result in the exposure of $\mathrm{BH} 3$ domain, leading to gain-offunctions for promoting apoptosis (Fig. 6D). Strikingly, we found that $\mathrm{N}$-terminal cleaved product of Beclin 1 (D150-450) alone is sufficient to induce cell death independent of any apoptotic stimulation (Fig. 5B). It is possible that exposure of the $\mathrm{BH} 3$ domain of Beclin 1 after cleavage by caspase- 3 may enhance apoptosis through direct binding to other antiapoptotic members in the Bcl-2 family and allow proapoptotic $\mathrm{BH} 3-$ only molecules to compete the Beclin 1 binding to $\mathrm{Bcl}-2 /$ $\mathrm{Bcl}-\mathrm{xL}$, resulting in apoptosis. Taken together, our data show that the interaction between $\mathrm{Bcl}-2$ and Beclin 1 is important for regulating autophagy and apoptosis. The cleavage of Beclin 1 by caspase disrupts the interaction between $\mathrm{Bcl}-2$ and Beclin 1, which can be prevented by z-VAD. Interestingly, although $\mathrm{D} 150-450$, the $\mathrm{N}$-terminal product of cleaved Beclin 1, was able to weakly bind to Bcl-2, D1-149 fails to interact with $\mathrm{Bcl}-2$. It is also unexpected that the expression of the non-cleavable mutant was unable to block the cleavage by caspases and it was sensitive to cell death in Beclin $1 \mathrm{KD}$ cells (Fig. 6). It is unexpected that the interaction between noncleavable Beclin1 mutant and $\mathrm{Bcl}-2$ were diminished
(Fig. 5B), likely due to these sites mutations are close the sites of its interaction with Bcl-2 (Maiuri et al., 2007b; Oberstein et al., 2007).

The precise relationship of autophagy and cell death is a subject of intense debate in recent years. Simultaneous activation of both autophagy and apoptosis are observed in many systems. For example, activation of TrkA and DAPK can lead to both apoptosis and autophagy (Gozuacik et al., 2008; Dadakhujaev et al., 2009; Zalckvar et al., 2009), but how autophagy, a proposed cell survival mechanism, contributing to apoptosis is not fully understood. Previous studies suggest that apoptosis induced by Bax blocks Beclin 1 dependent autophagy through the cleavage of Beclin 1 (Luo and Rubinsztein, 2009). Our study concludes that Beclin 1 cleavage by caspase-3 promotes apoptosis through gain-offunctions in addition its role to inactivate autophagy as previous suggested. In conclusion, our data suggest that autophagy is one of the early events following treatment with death stimuli and inactivation of autophagy by cleavage of Beclin 1 leads to apoptosis. Our findings offer new insights for understanding the interplay of autophagy and apoptosis.

\section{MATERIALS AND METHODS}

\section{Materials}

Anti- $\beta$-actin monoclonal and anti LC3 antibody (Sigma A5441, L7543), anti-cytochrome $c$ antibody (BD, 556432 for $W B$ and 556433 for IP), anti-Beclin 1 (BD 612113) and anti-Bcl-2 (BD 610539); anti-caspase-3 (Biomol and Produced by $Q$ Chen 

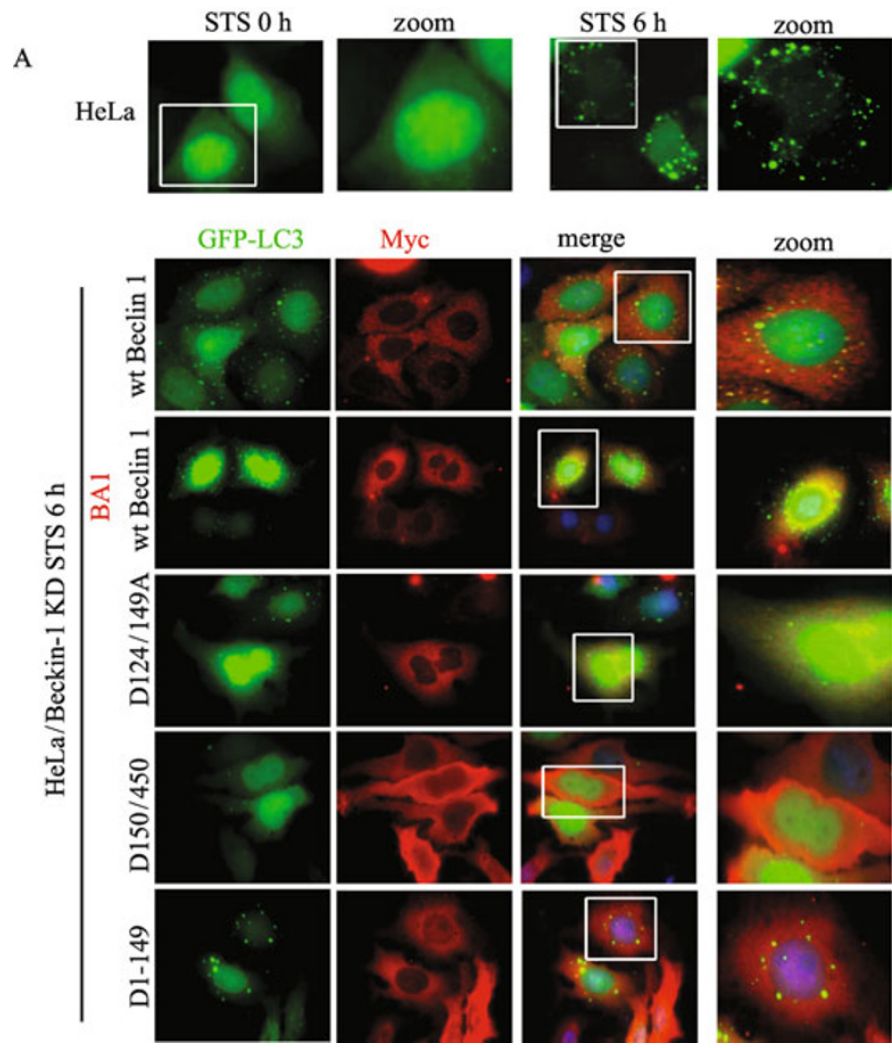

merge
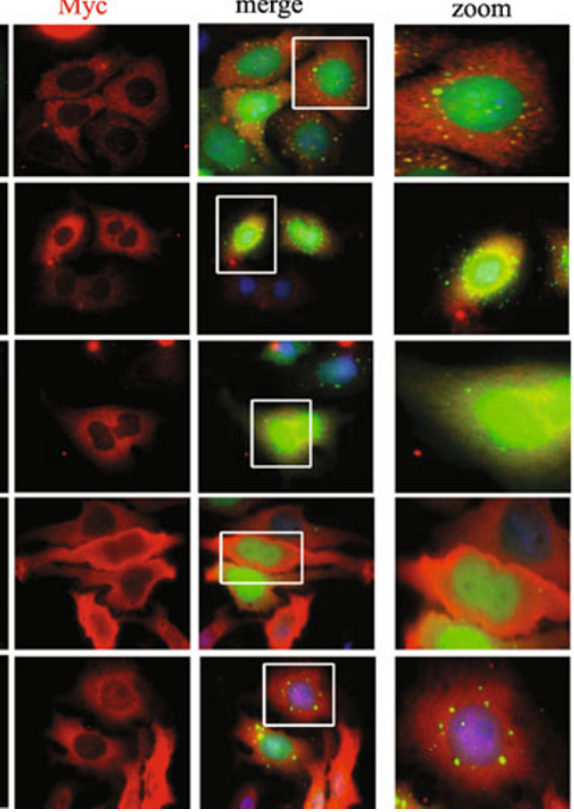

STS $12 \mathrm{~h}$

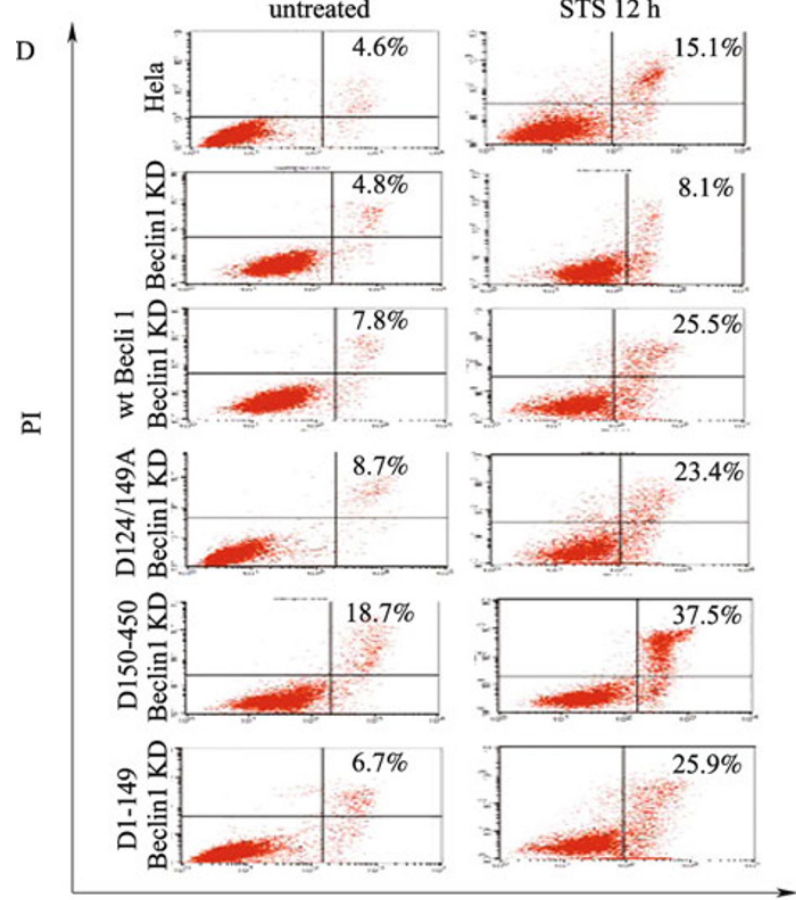

FITC-Annexin V
B

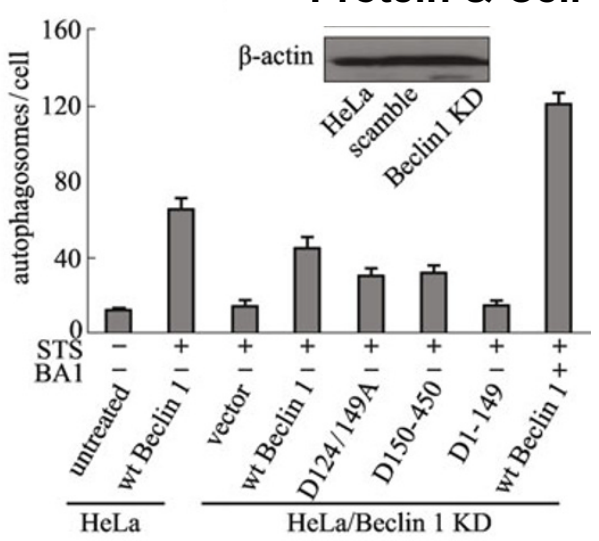

C

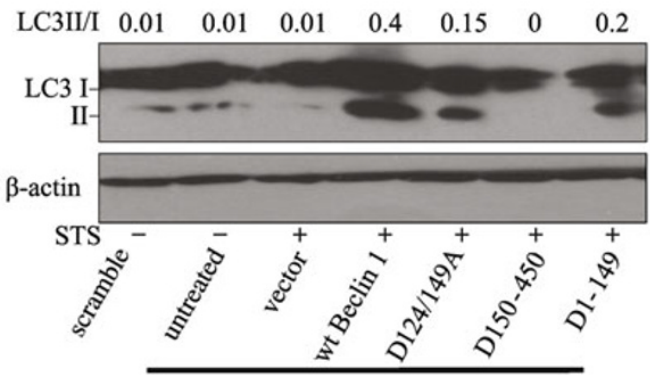

$\mathrm{HeLa} / \mathrm{Beclin} 1 \mathrm{KD}$

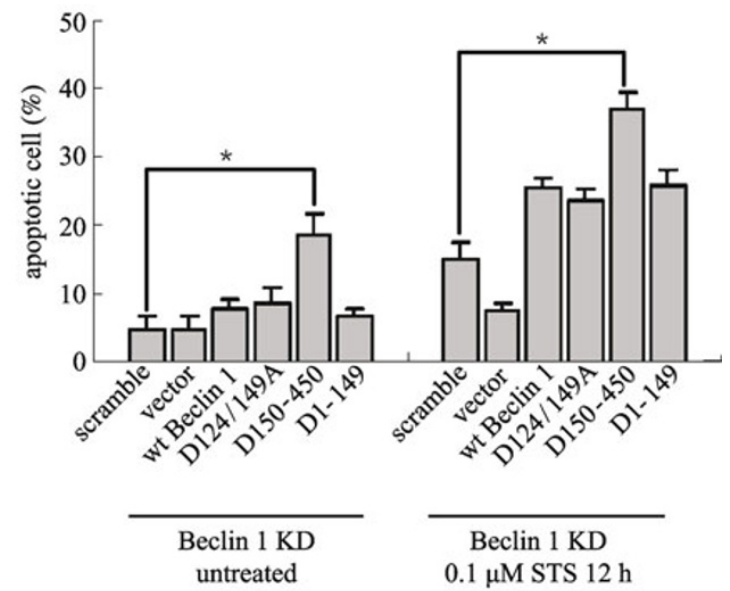

Figure 6. The effect of wild type Beclin 1 and its mutants or truncations on autophagy and apoptosis in Beclin 1 knocked-down cells. (A, B) HeLa cells were transfected with the pSilencer ${ }^{\mathrm{TM}}$ 4.1-CMV/neo vector that contains a 19-mer hairpin sequence specific to the Beclin 1 mRNA target and stable cell line were selected by G418. Beclin 1 protein levels in KD cells were confirmed by anti-Beclin 1 antibody. Measurement of the autophagosome formation in Beclin $1 \mathrm{KD}$ cells (A, B). GFP-LC3 was co-transfected with Beclin 1 or its mutants or truncations (1:1 ratio) in Beclin $1 \mathrm{KD}$ cells for $24 \mathrm{~h}$ and then treated with staurosporine for $6 \mathrm{~h}$ in the absence or presence of BA1 $(20 \mathrm{nM})$. Cells were fixed with formaldehyde $(3.7 \%, w / v)$ and immuostained with anti-myc for Beclin 1 and GFP for autophagosomes by fluoromicroscopy. Statistical results shown in B and data were mean value of at least 3 independent experiments. (C) WT Beclin 1 or its mutants or truncations were transfected in Beclin $1 \mathrm{KD}$ cells for $24 \mathrm{~h}$, and then treated by staurosporine $(0.1 \mu \mathrm{M})$ for $6 \mathrm{~h}$ before detection of LC3 levels by Western blotting. (D) WT Beclin 1 or its mutants or truncations were transfected into Beclin $1 \mathrm{KD}$ cells for $24 \mathrm{~h}$, and then treated by $0.1 \mu \mathrm{M}$ staurosporine for another $24 \mathrm{~h}$ before detection of cell death by flow cytometry after the staining of PI and Annexin V. Data were mean value of at least 3 independent experiments with duplicate samples. ${ }^{*} p<0.05$ compared with the control cells. 
Laboratory), anti-lamp3 (SC-5275), anti-myc (SC-40)a, caspase inhibitor z-VAD-fmk (CalBiochem 627610), CaspACETM FITC-VADfmk (Promega G7462). Secondary antibodies (HRP-labeled Goat Anti-Mouse IgG, 074-1806, HRP-labeled Goat Anti-Rabbit IgG, 0741506) and enhanced chemiluminescence (ECL, WBKLS0500) reagents were purchased from KPL and Millipore, respectively. All other chemicals were purchased from Sigma unless otherwise specified.

\section{Cell culture and transfection}

Cell cultures were maintained in DMEM medium supplemented with $10 \%$ FBS (Hyclone) and $1 \%$ penicillin-streptomycin at $37^{\circ} \mathrm{C}$ and $5 \%$ $\mathrm{CO}_{2}$. To establish cell lines stably expressing Beclin 1, HeLa cells were transfected with pCDNA4.0-Beclin 1/myc.his plasmid DNA by phosphate calcium method, and positive clones overexpressing Beclin 1 were selected with $100 \mu \mathrm{g} / \mathrm{mL}$ Zoecin (Invitrogen R250-01). For serum and amino-acid starvation, cells were cultured in serumfree Earle's Balanced Salt Solution medium (Gibco).

\section{Detection of cell death by Hoechst 33342 and Annexin-V FITC}

For Hoechst 33342 staining, cells were plated at low density on glass coverslipes in a six-well plate and treated with staurosporine. At the indicated times, cells were stained with Hoechst 33342 in PBS (15 min at room temperature in the dark). Cells were then washed three times with PBS and analyzed under a fluorescence microscope, with at least 200 cells counted. Apoptotic cells were detected by flow cytometry. Phosphatidylserine exposed on the outside of the cells was determined by Annexin V-FITC staining. Briefly, cells were plated in six-well plates and treated with staurosporine for the indicated time. At the end of the experiment, cells were digested with Trypsin-EDTA solution, then collected by centrifugation and washed twice with icecold PBS. After being washed one more time with the reaction buffer (10 mM HEPES/NaOH, pH 7.4, $140 \mathrm{mM} \mathrm{NaCl}$, and $5 \mathrm{mM} \mathrm{CaCl}_{2}$ ), cells were stained with Annexin V-FITC for $10 \mathrm{~min}$ in the dark at room temperature; then an additional $100 \mu \mathrm{L}$ binding buffer and $5 \mu \mathrm{L} 50 \mu \mathrm{M}$ PI were added before FACS analysis. Flow cytometric analysis was performed to monitor the green fluorescence of the FITC-conjugated Annexin V $(530 \pm 30 \mathrm{~nm})$ and the red fluorescence of DNA-bound PI $(630 \pm 22 \mathrm{~nm})$. All data was analyzed with Cell Quest software (BD).

\section{Detection of caspase activation in situ}

The method was performed following the CaspACETM FITC-VADfmk in situ Marker kit manual. Briefly, cells were digested with trypsinEDTA solution, then collected and washed with PBS. CaspACETM FITC-VAD-fmk in situ Marker was added to the cells (100 $\mu \mathrm{L}$ DMEM containing $10^{5}$ cells) at a final concentration of $10 \mu \mathrm{M}$, and then incubated for $20 \mathrm{~min}$ in the dark the supplemented with $100 \mu \mathrm{L}$ DMEM and measured with a FACS Scan.

\section{SDS-PAGE and Western blotting}

SDS-PAGE and western blotting were performed as described previously. Briefly, the cells or membrane fractions were resuspended in NP-40 containing lysis buffer (10 mM HEPES $(\mathrm{pH} 7.4), 2 \mathrm{mM}$ EGTA, $0.5 \%$ NP-40, $1 \mathrm{mM} \mathrm{NaF}, 1 \mathrm{mM} \mathrm{NaVO} 4,1 \mathrm{mM}$ phenylmethyl- sulfonyl fluoride (PMSF), $1 \mathrm{mM} \mathrm{DTT,} 50 \mathrm{mg} / \mathrm{mL}$ trypsin inhibitor, 10 $\mathrm{mg} / \mathrm{mL}$ aprotinin, and leupeptin) and placed on ice for $30 \mathrm{~min}$. The lysates were centrifuged at $12,000 \times g$ for $12 \mathrm{~min}$ at $4^{\circ} \mathrm{C}$, and the protein concentration was determined with BSA as a standard. Equivalent samples $(20 \mu \mathrm{g}$ protein) were subjected to SDS-PAGE on $12 \%$ gel. The proteins were then transferred onto nitrocellulose membranes, and probed with the indicated antibodies followed by the appropriate secondary antibodies conjugated to horseradish peroxidase (KPL, Gaithersburg, MD, USA). Immunoreactive bands were visualized using enhanced chemiluminescence (Pierce).

\section{Co-Immunoprecipitation experiment}

HeLa cells were transiently transfected using the phosphate calcium method with pcDNA4TO/Myc.His plasmids expressing Myc.Histagged Beclin 1, alone or together with HA-tagged Bcl-2. Twentyfour hours later, cells were collected and lysed in NP40 buffer $(50 \mathrm{mM}$ HEPES pH 7.4, $150 \mathrm{mM} \mathrm{NaCl}, 10 \%$ glycerol, $1 \mathrm{mM}$ EDTA and $10 \mathrm{mM}$ $\mathrm{PMSF})$. The clear lysates were then subjected to Co-Immunoprecipitation with anti-myc or anti-Bcl-2 for binding over-night at $4^{\circ} \mathrm{C}$. Then the Protein-G agrose was added for binding for $2 \mathrm{~h}$. The immunoprecipitates were analyzed by lotting with anti-Bcl-2 or anti-Beclin 1 antibody.

\section{Analysis of Autophagosomes}

GFP-LC3/HeLa cells cultured on coverslips were fixed with formaldehyde $(3.7 \%, w / v)$ and stained with Anti-myc for Beclin 1 expression and Hoechst $33342(2 \mu \mathrm{M}$, Sigma). Cells presenting a mostly diffuse distribution of GFP-LC3 in the cytoplasm and nucleus were considered as non-autophagic, whereas cells representing several intense punctuate GFP-LC3 aggregates with no nuclear GFP-LC3 were classified as autophagic.

\section{Cleavage of Beclin 1 protein}

The caspase in vitro cleavage experiment was performed as described previously with modification. In brief, $20 \mu \mathrm{g}$ of cell lysate was incubated with $0-200 \mathrm{ng}$ of recombinant caspase- 3 at $37^{\circ} \mathrm{C}$ for $2 \mathrm{~h}$ in $50 \mu \mathrm{L}$ of reaction buffer ( $100 \mathrm{mM}$ Hepes $\mathrm{pH} 7.5,20 \%$ glycerol, $0.5 \mathrm{mM}$ EDTA, $10 \mathrm{mM}$ DTT). The reactions were stopped with addition of loading buffer and boiled for $5 \mathrm{~min}$, resolved by $12 \%$ SDS-PAGE and immunblotted with Beclin 1 antibody.

\section{ACKNOWLEDGMENTS}

We thank Drs. Li Yu and Aimin Zhou for their critical reading of the manuscript. This work was supported by the National Basic Research Program (973 program project) (grants Nos. 2007CB914800, 2006СВ910102), grants from National Natural Science Foundation of China (grant Nos. 30630038 and 30400098), grants from Tianjin Natural Science foundation (09JCZDJC21200) to Y. Zhu and a project grant from Chinese Academy of Sciences KSCX2-YW-R-02 to Q. Chen.

\section{REFERENCES}

Cho, D.H., Jo, Y.K., Hwang, J.J., Lee, Y.M., Roh, S.A., and Kim, J.C. 
(2009). Caspase-mediated cleavage of ATG6/Beclin-1 links apoptosis to autophagy in HeLa cells. Cancer Lett 274, 95-100.

Codogno, P., and Meijer, A.J. (2005). Autophagy and signaling: their role in cell survival and cell death. Cell Death Differ 12, 1509-1518.

Dadakhujaev, S., Jung, E.J., Noh, H.S., Hah, Y.S., Kim, C.J., and Kim, D.R. (2009). Interplay between autophagy and apoptosis in TrkAinduced cell death. Autophagy 5.

Eisenberg-Lerner, A., Bialik, S., Simon, H.U., and Kimchi, A. (2009). Life and death partners: apoptosis, autophagy and the cross-talk between them. Cell Death Differ 16, 966-975.

Feng, W., Huang, S., Wu, H., and Zhang, M. (2007). Molecular basis of Bcl-xL's target recognition versatility revealed by the structure of $\mathrm{Bcl}-\mathrm{xL}$ in complex with the BH3 domain of Beclin-1. J Mol Biol 372, 223-235.

Gozuacik, D., Bialik, S., Raveh, T., Mitou, G., Shohat, G., Sabanay, H., Mizushima, N., Yoshimori, T., and Kimchi, A. (2008). DAPkinase is a mediator of endoplasmic reticulum stress-induced caspase activation and autophagic cell death. Cell Death Differ 15, 1875-1886.

Kroemer, G., and Jaattela, M. (2005). Lysosomes and autophagy in cell death control. Nat Rev Cancer 5, 886-897.

Levine, B., and Kroemer, G. (2008). Autophagy in the pathogenesis of disease. Cell 132, 27-42.

Levine, B., and Kroemer, G. (2009). Autophagy in aging, disease and death: the true identity of a cell death impostor. Cell Death Differ $16,1-2$.

Luo, S., and Rubinsztein, D.C. (2009). Apoptosis blocks Beclin 1dependent autophagosome synthesis: an effect rescued by Bcl-xL. Cell Death Differ 17, 268-277.

Maiuri, M.C., Criollo, A., Tasdemir, E., Vicencio, J.M., Tajeddine, N., Hickman, J.A., Geneste, O., and Kroemer, G. (2007a). BH3-only proteins and $\mathrm{BH} 3$ mimetics induce autophagy by competitively disrupting the interaction between Beclin 1 and $\mathrm{Bcl}-2 / \mathrm{Bcl}-\mathrm{X}(\mathrm{L})$. Autophagy 3, 374-376.

Maiuri, M.C., Le Toumelin, G., Criollo, A., Rain, J.C., Gautier, F., Juin, P., Tasdemir, E., Pierron, G., Troulinaki, K., Tavernarakis, N., et al. (2007b). Functional and physical interaction between $\mathrm{Bcl}-\mathrm{X}(\mathrm{L})$ and a BH3-like domain in Beclin-1. EMBO J 26, 2527-2539.

Maiuri, M.C., Zalckvar, E., Kimchi, A., and Kroemer, G. (2007c).
Self-eating and self-killing: crosstalk between autophagy and apoptosis. Nat Rev Mol Cell Biol 8, 741-752.

Oberstein, A., Jeffrey, P.D., and Shi, Y. (2007). Crystal structure of the Bcl-XL-Beclin 1 peptide complex: Beclin 1 is a novel BH3-only protein. J Biol Chem 282, 13123-13132.

Orvedahl, A., and Levine, B. (2008). Autophagy and viral neurovirulence. Cell Microbiol 10, 1747-1756.

Pattingre, S., and Levine, B. (2006). Bcl-2 inhibition of autophagy: a new route to cancer? Cancer Res 66, 2885-2888.

Pattingre, S., Tassa, A., Qu, X., Garuti, R., Liang, X.H., Mizushima, N., Packer, M., Schneider, M.D., and Levine, B. (2005). Bcl-2 antiapoptotic proteins inhibit Beclin 1-dependent autophagy. Cell 122, 927-939.

Pattingre, S., Bauvy, C., Carpentier, S., Levade, T., Levine, B., and Codogno, P. (2009). Role of JNK1-dependent Bcl-2 phosphorylation in ceramide-induced macroautophagy. J Biol Chem 284, 2719-2728.

Shimizu, S., Kanaseki, T., Mizushima, N., Mizuta, T., ArakawaKobayashi, S., Thompson, C.B., and Tsujimoto, Y. (2004). Role of $\mathrm{Bcl}-2$ family proteins in a non-apoptotic programmed cell death dependent on autophagy genes. Nat Cell Biol 6, 1221-1228.

Wang, J. (2008). Beclin 1 bridges autophagy, apoptosis and differentiation. Autophagy 4, 947-948.

Wei, Y., Pattingre, S., Sinha, S., Bassik, M., and Levine, B. (2008a). JNK1-mediated phosphorylation of $\mathrm{Bcl}-2$ regulates starvationinduced autophagy. Mol Cell 30, 678-688.

Wei, Y., Sinha, S., and Levine, B. (2008b). Dual role of JNK1mediated phosphorylation of $\mathrm{Bcl}-2$ in autophagy and apoptosis regulation. Autophagy 4, 949-951.

Yorimitsu, T., and Klionsky, D.J. (2005). Autophagy: molecular machinery for self-eating. Cell Death Differ 12, 1542-1552.

Yousefi, S., Perozzo, R., Schmid, I., Ziemiecki, A., Schaffner, T., Scapozza, L., Brunner, T., and Simon, H.U. (2006). Calpainmediated cleavage of Atg5 switches autophagy to apoptosis. Nat Cell Biol 8, 1124-1132.

Zalckvar, E., Berissi, H., Mizrachy, L., Idelchuk, Y., Koren, I., Eisenstein, M., Sabanay, H., Pinkas-Kramarski, R., and Kimchi, A. (2009). DAP-kinase-mediated phosphorylation on the BH3 domain of beclin 1 promotes dissociation of beclin 1 from $\mathrm{Bcl}-\mathrm{XL}$ and induction of autophagy. EMBO Rep 10, 285-292. 